\title{
Aprendizaje basado en equipos con IF-AT: Impacto y percepción en estudiantes universitarios
}

\author{
Team Based Learning with IF-AT: Impact and perception in university students
Aprendizagem baseada em equipamentos com IF-AT: Impacto e percepção em estudantes universitários

\author{
Sofía Ruiz-Campo ${ }^{1}$, Karla Soria-Barreto ${ }^{2}$, Sergio Zúñiga-Jara ${ }^{3}$ \\ ${ }^{1}$ Universidad Católica del Norte, Chile, (56-51) 220 9843, sruizcampo@ucn.cl \\ 2Universidad Católica del Norte, Chile, (56-51) 220 9843, ksoria@ucn.cl \\ ${ }^{3}$ Universidad Católica del Norte, Chile, (56-51) 220 9843, sz@ucn.cl
}

\begin{abstract}
RESUMEN
En este estudio se analizan los resultados de implementar una metodología activa de enseñanza denominada Aprendizaje Basado en Equipos (TBL) en una asignatura de una universidad tradicional chilena. Para ello se aplicó un instrumento de recopilación de información a dos grupos comparables de estudiantes durante un semestre. En un grupo se utilizó la metodología tradicional basada en la clase magistral, y al otro grupo se le aplicó la técnica TBL. Como resultado, las pruebas estadísticas mostraron evidencia de que los estudiantes lograron un mejor desempeño académico y un mayor aprendizaje bajo esta nueva metodología. Adicionalmente, los estudiantes generalmente mostraron un mayor interés por la materia, participación más activa en las clases, y la sensación de que el proceso de enseñanza-aprendizaje era más efectivo.
\end{abstract}

Palabras clave: aprendizaje basado en equipos, metodología activa, IF-AT.

\section{ABSTRACT}

This study discusses the results of implementing an active teaching methodology called Team Based Learning (TBL) in a subject of the Business Administration Career, in a Chilean traditional university. A data collection instrument was applied to two groups of students during a semester. The traditional lecture class-based methodology was used in a group, and in the other group the TBL technique was applied. As a result, the statistical tests showed evidence that students achieved better academic performance and a higher learning under this new methodology. Additionally, students generally showed a greater interest in the course subject matter, more active participation in classes, and the feeling that the teaching-learning process was more effective.

Keywords: team based learning, active methodology, IF-AT.

\section{RESUMO}

Analisaram-se os resultados da implementação de uma metodologia ativa de ensino denominada Aprendizagem Baseada em Equipamentos (TBL) em uma disciplina de uma universidade tradicional do Chile. Para tanto, aplicou-se um instrumento de compilação de informação a dois comparáveis grupos de estudantes durante um semestre. Para um dos grupos, empregou-se a metodologia tradicional baseada na aula expositiva e, ao outro, foi empregada a técnica TBL. Como resultado, as provas estatísticas evidenciaram que os estudantes do segundo grupo alcançaram melhores resultados e maior aprendizagem. Além do mais, os estudantes, em geral, mostraram maior interesse pela matéria, maior participação ativa nas aulas e sensação de que o processo de ensinoaprendizagem era mais efetivo.

Palavras-chave: aprendizagem baseada em equipamentos, metodologia ativa, IF-AT. 


\section{INTRODUCCIÓN}

En años recientes, la mayor parte de los centros de educación superior chilenos han impulsado nuevos currículos basados en el desarrollo de competencias y en el uso de nuevas metodologías de enseñanza-aprendizaje (Moreno-Olivos, 2010; Salgado et al., 2012; Asún et al., 2012).

La docencia universitaria tradicional suele basarse en la cátedra magistral, la que requiere de estudiantes entrenados, motivados y responsables, que preparan anticipadamente las materias para exámenes solemnes, generalmente tomados dos veces durante un semestre. Como una forma de mantener a los estudiantes trabajando durante el semestre, se suele aplicar controles de lectura, talleres y otros sistemas de evaluación intermedias, lo que suele resultar difícil en cursos numerosos, por el incremento de carga sobre académicos y ayudantes. Sin embargo, a fines de los años 70 surgió la estrategia pedagógica denominada Aprendizaje Basado en Equipos (TBL, "Team Based Learning”), desarrollada por Larry Michaelsen, de la Universidad de Oklahoma. El objetivo fue cambiar la forma de aprendizaje pasiva por una más activa, trabajando en conjunto e interactuando. El aprendizaje mejora por la transferencia de habilidades de unos estudiantes a otros, a través de la resolución conjunta de problemas.

Michaelsen et al. (2002) señalan que el TBL es más efectivo para crear un cuerpo de conocimiento utilizable en el futuro, debido a que las habilidades profesionales más importantes están referidas a la resolución de problemas, más que a la memorización. En el caso de Chile, se dispone de la experiencia de Lee et al. (2014) para la carrera de odontología de la Universidad de Chile. Recientemente, la técnica se ha ido extendiendo a otras áreas del conocimiento fuera de la medicina (Polimeno et al., 2012; Abbott, 2012; Clark et al., 2008; Nieder et al., 2005).

El proceso de TBL está bien descrito por Michaelsen y Sweet (2008), quienes indican que la creación e implementación de tareas efectivas en grupos, siguiendo TBL, se divide en tres partes (Figura 1). Durante la pre-clase, el trabajo se centra en el individuo, según una tarea previamente establecida por el profesor. En la clase, los estudiantes se organizan en grupos para aplicar los contenidos mediante discusión en equipo. Finalmente, durante la clase los estudiantes reciben retroalimentación continua e inmediata.

Fig. 1. Composición de la enseñanza según le metodología TBL

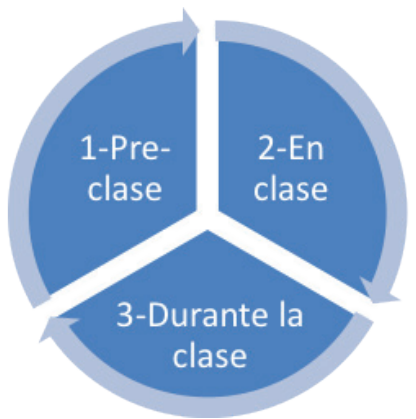

Fuente: Elaboración propia, con conceptos de Michaelsen y Sweet (2008). 
La secuencia mostrada en la Figura 2 ilustra las fases del TBL. La fase 1 incluye el trabajo o estudio individual pre-clase; la fase 2 incorpora el test individual, la discusión por grupos, la discusión de la clase y la retroalimentación por parte del profesor; y la tercera fase supone una explicación de conceptos y el envío de tareas, ya que los estudiantes rinden cuentas, tanto al instructor como a sus compañeros de equipo, por la calidad y la cantidad de su trabajo individual.

Fig. 2. Secuencia de actividades en el Aprendizaje Basado en Equipos (TBL)

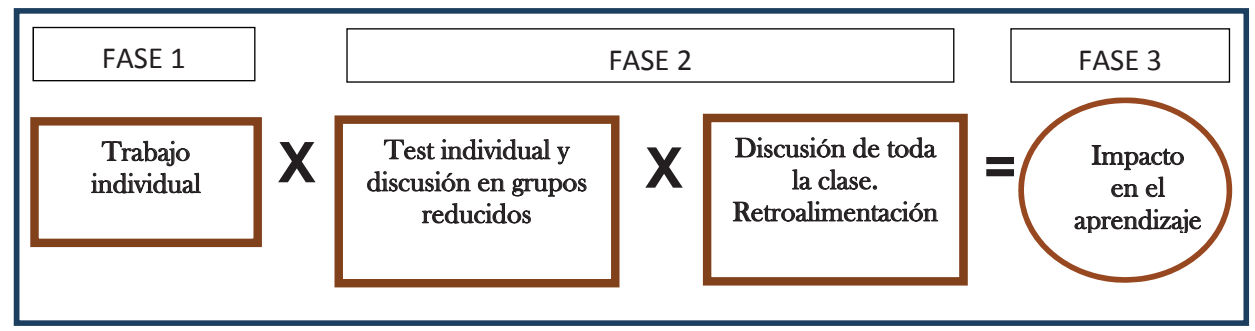

Fuente: Adaptación de Michaelsen y Sweet (2008).

Para un aprendizaje efectivo, las tareas de un sistema TBL deben ser diseñadas en torno a un problema que es significativo para los estudiantes; todos los estudiantes deben estar trabajando en el mismo problema haciendo una elección específica (sólo una solución posible), y los grupos deben informar al mismo tiempo sobre sus opciones.

La formación de grupos permite aprender a trabajar en equipo, dialogar, negociar y aportar ideas (Brodbeck et al., 2002). Se pueden conformar grupos pequeños, de no más de 3 integrantes, designados aleatoriamente en cada sesión. Con el TBL, el estudiante deja de ser un receptor pasivo y se transforma en el artífice principal de su aprendizaje, autogestionando su tiempo de estudio para llevar los contenidos preparados a las sesiones de trabajos grupales. El trabajo en equipo supone una exigencia social, ya que si algún estudiante no lleva preparada la clase, esto va a repercutir en el desempeño de sus compañeros de grupo, y va a ser juzgado también por ellos (Lerner y Tetlock, 1999).

Cuando el TBL es implementado junto con la técnica IF-AT ("Immediate Feedback Assessment Technique"), la evaluación se hace aún más interactiva e inmediata. La técnica IF-AT se basa en una tarjeta con formato de respuesta de selección múltiple (Figura 3). Los estudiantes raspan una alternativa, y si ésta es correcta aparece una estrella, y en caso contrario no aparece nada y deben hacer otro intento. Si el IF-AT es devuelta por los grupos de estudiantes al profesor con sólo una alternativa raspada en cada pregunta, se obtendrá el puntaje máximo del test. Se aplica un descuento de puntaje mientras más alternativas raspadas existan en cada pregunta. El elemento relevante es que los grupos apenas acuerdan realizar un raspe obtienen retroalimentación, y surge una discusión inmediata en el interior de cada grupo. 
Fig. 3. Tarjetas IF-AT sin uso (izquierda) y con respuestas (centro y derecha)

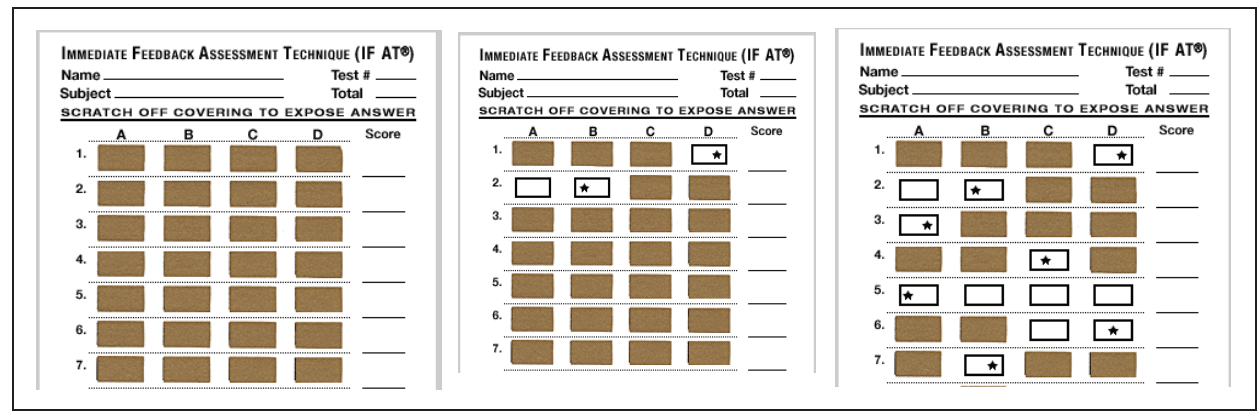

Fuente: Epstein (1989).

El TBL requiere la preparación previa del material del curso por parte del docente, y en esto, el apoyo de ayudantes entrenados es fundamental. La mayor parte del trabajo del profesor no está ahora en preparar la clase magistral, sino en preparar lecturas y formular problemas en formato de selección múltiple.

En resumen, en comparación con las técnicas tradicionales de enseñanza, la técnica TBL requiere mayor responsabilidad del estudiante, un trabajo más continuado, una preparación pre-clase y en clase, y una retroalimentación inmediata (Tabla 1).

Tabla 1. Comparativa de las características de las técnicas Tradicional y TBL

\begin{tabular}{|c|c|c|}
\hline Características & $\begin{array}{l}\text { Metodología Tradicional: } \\
\text { clase magistral }\end{array}$ & Metodología TBL \\
\hline Pre-clase & $\begin{array}{c}\text { El estudiante podría estudiar } \\
\text { los conceptos antes de cada } \\
\text { clase. }\end{array}$ & $\begin{array}{l}\text { El estudiante debe estudiar los conceptos } \\
\text { para el test. }\end{array}$ \\
\hline En la clase & $\begin{array}{l}\text { El profesor presenta la clase } \\
\text { magistral, y el estudiante } \\
\text { toma notas pasivamente. }\end{array}$ & $\begin{array}{l}\text { El estudiante es evaluado y aplica los } \\
\text { conocimientos que ha preparado. Se une } \\
\text { activamente a equipos para resolver tests. }\end{array}$ \\
\hline $\begin{array}{l}\text { Responsabilidad del } \\
\text { estudiante }\end{array}$ & $\begin{array}{l}\text { Prepararse para los } \\
\text { exámenes. }\end{array}$ & $\begin{array}{l}\text { Realizar trabajo pre-clase, trabajar clases } \\
\text { individualmente y en equipo. }\end{array}$ \\
\hline $\begin{array}{l}\text { Resultados de las } \\
\text { pruebas }\end{array}$ & $\begin{array}{c}\text { Días o semanas después de } \\
\text { las pruebas. }\end{array}$ & Retroalimentación inmediata. \\
\hline $\begin{array}{l}\text { Los objetivos a final } \\
\text { de curso }\end{array}$ & $\begin{array}{l}\text { Conocer la materia y } \\
\text { resolver problemas. }\end{array}$ & $\begin{array}{c}\text { Conocer la materia y resolver problemas. } \\
\text { Desarrollar la capacidad de trabajo } \\
\text { colaborativo. Lograr un aprendizaje } \\
\text { duradero. }\end{array}$ \\
\hline
\end{tabular}

Fuente: Elaboración propia. 
Además, el TBL parece resultar útil no sólo para cursos pequeños de estudiantes, sino también para cursos numerosos, e incluso en materias en las que se requiere realizar cálculos numéricos de relativa complejidad. Evaluar lo anterior, a través de un caso de estudio, es el objetivo central de la presente investigación.

\section{APLICACIÓN DEL TBL/IF-AT EN CURSOS UNIVERSITARIOS NUMEROSOS}

El objetivo de este estudio es estimar si la implementación de la metodología de TBL genera impactos positivos o deseables en el aprendizaje en cursos relativamente numerosos de estudiantes universitarios. Para ello se proponen tres hipótesis:

Hipótesis 1: La metodología TBL logra mejores resultados, es decir, la nota final de aprobación de la asignatura es mayor que la obtenida con la metodología de cátedra tradicional.

Los estudios realizados en relación a la implementación del TBL detallan que el aprendizaje logrado por los estudiantes es significativamente mejor (Polimeno et al., 2012, Abbott, 2012; Clark et al., 2008; y Nieder et al., 2005). En este sentido, interesa verificar si luego de realizar un curso con TBL, los estudiantes obtuvieron una nota final del curso significativamente mayor desde el punto de vista estadístico, en comparación a la obtenida por estudiantes que cursaron la asignatura con la metodología tradicional.

Hipótesis 2: Los estudiantes tienen una mejor percepción de la metodología TBL como herramienta de aprendizaje que la metodología tradicional.

Michaelsen y Sweet (2008) afirman que los estudiantes logran autogestionar mejor su aprendizaje a través de la metodología del TBL, ya que la metodología activa los motiva y favorece su proceso de enseñanza-aprendizaje. En concreto, se busca comprobar que los estudiantes efectivamente tienen una mejor percepción de la metodología TBL comparada con la clase magistral como medio para lograr el aprendizaje.

Hipótesis 3: Mayores notas de enseñanza media (NEM) favorecen el aprendizaje a través de la metodología de TBL.

Esta hipótesis tiene base en estudios que señalan que aquellos estudiantes con mayores NEM tienen mejores hábitos de estudio, y son más responsables en su proceso de aprendizaje (Duarte y Galaz, 2006; Chain et al., 2003 y Soria-Barreto y Zúñiga-Jara, 2014). Interesa verificar entonces si el buen rendimiento escolar en la enseñanza media favorece o no los resultados de la metodología TBL.

Como objeto de estudio se ha escogido una cátedra del área de finanzas de la carrera de Ingeniería Comercial de una gran universidad tradicional chilena. Para ello, se han considerado dos grupos de estudiantes. El primer grupo (de control), con estudiantes que cursaron la asignatura en semestres previos utilizando la técnica docente tradicional (clase magistral). El segundo grupo lo constituyen estudiantes que cursaron la misma asignatura, con el mismo profesor, pero usando la metodología TBL.

En la metodología tradicional, el curso tiene una duración de un semestre, con una carga de 5 créditos, distribuidos en 3 horas directas de cátedra semanal presenciales con 
el profesor, 3 horas indirectas semanales que el estudiante dedica al estudio, y 1,5 horas directas de práctica semanal de clases presenciales con el ayudante. Las horas directas de cátedra semanal eran organizadas en dos sesiones por semana, de una duración de 1,5 horas cada una. El curso fue evaluado a través de una serie de trabajos prácticos, dos exámenes parciales, y un examen final.

En la implementación del TBL, se realizó sólo una sesión por semana, en dos bloques seguidos de noventa minutos, con un descanso de 15 minutos entre los dos bloques (Figura 4). Se requirió una sala provista de mesas trapecio individuales de $1.20 \times 0.60 \mathrm{~m} \times 0.60 \mathrm{~m}$, las que permiten ser movilizadas para formar grupos de estudiantes de distinto tamaño.

En el primer bloque de clases se aplicó el test individual de aproximadamente 30 minutos (RAP, "Readiness Assurance Process" o Proceso de Aprendizaje Inicial). Luego, los estudiantes se organizaron en grupos de un modo aleatorio proporcionado por el profesor. A cada grupo se le entregó una tarjeta IF-AT para volver a realizar el test previo, pero grupalmente.

Al inicio del segundo bloque, las notas fueron entregadas a los estudiantes. Sin embargo, al terminar el primer bloque los estudiantes ya tenían una expectativa bastante acertada de cuál sería su nota. Los estudiantes solicitaban aclarar dudas del test, y el profesor identificaba las áreas a reforzar, basándose también en los resultados del test. A continuación, se entregaba a los estudiantes un breve cuestionario llamado "One Minute Paper" para que respondieran por escrito y anónimamente, si lo desean, a dos o tres preguntas breves sobre el ciclo de aprendizaje semanal que acababa de terminar (Lazerson et al., 1999; Angelo y Cross, 1993). Las preguntas típicas son ¿qué ha sido para tí lo más importante que has aprendido en esta semana? (o lo que más te gustó, etc.), ¿qué es lo que te ha quedado más confuso? y ¿qué recomendación harías al profesor y ayudantes para mejorar tu aprendizaje? Esta información fue procesada posteriormente y se incorporó al aprendizaje.

Luego el profesor introdujo el tema a trabajar en la semana y que sería evaluado en la siguiente sesión, informando claramente los conceptos relevantes a estudiar, ofreciendo documentación, bibliografía, y guías de ejercicios resueltos de esta materia, todos en formato de selección múltiple. Finalmente el profesor realizó la clase expositiva acerca de tales materias, destacando los aspectos relevantes y resolviendo los problemas más complicados, para facilitar el trabajo a realizar por los estudiantes durante la semana, los que serían evaluadas en el primer bloque de la siguiente semana, comenzando así de nuevo el ciclo.

Fig. 4. Distribución del tiempo en una clase experimental con la metodología TBL

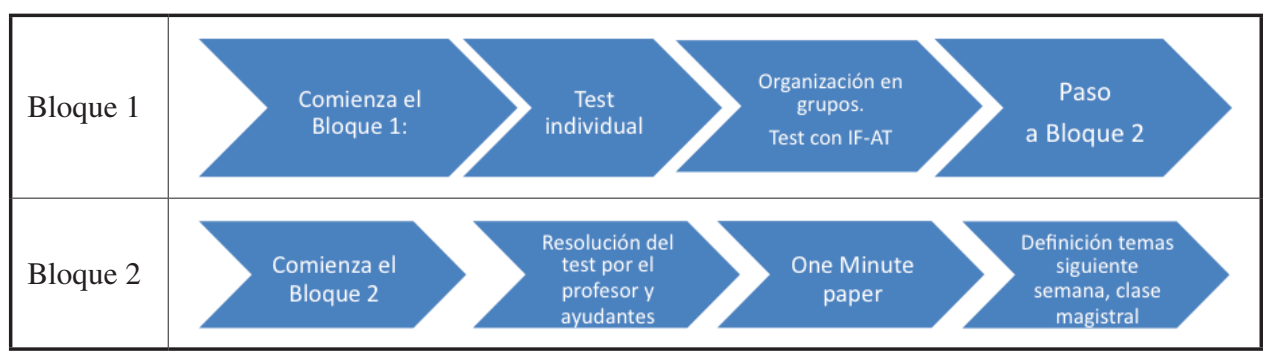

Fuente: Elaboración propia. 
Respecto a la forma de evaluación del curso, aquí también se introdujeron cambios significativos. En primer lugar, las pruebas parciales o solemnes se reemplazaron por las RAP semanales. También cambió la ponderación de las notas: el promedio de los tests individuales tuvo una ponderación del $40 \%$ en la nota final; el promedio de los tests grupales, el 10\%; y el examen global de toda la materia final, el 50\% (Figura 5). Resulta interesante resaltar que se fijó como requisito para aprobar el curso aprobar el examen final, independiente de las notas de los otros test. En los casos de inasistencias justificadas a los test, se calcula el promedio de los tests con n-1 notas, siendo n el número de test a considerar.

Fig. 5. Composición de la nota final del curso

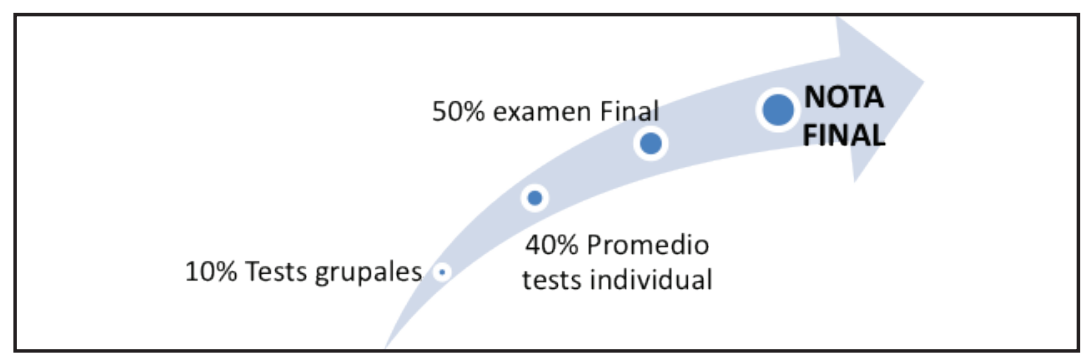

Fuente: Elaboración propia.

\section{LA ENCUESTA}

Se diseñó un cuestionario como instrumento de recopilación de información, el que fue aplicado a los dos grupos de estudiantes que cursaron la asignatura, ambos grupos con el mismo profesor, pero en dos semestres consecutivos. Cada grupo utilizó una metodología diferente, la tradicional y la basada en el TBL con IF-AT. El instrumento estaba compuesto por tres grupos de preguntas. La primera parte correspondía a datos generales del estudiante, tal como tipo de colegio de procedencia, nota de enseñanza media y nivel de educación de los padres. La segunda parte incluyó preguntas relacionadas con los antecedentes de la asignatura, tales como el semestre y año en que se cursó la asignatura, la oportunidad y nota final de la misma. La última parte de la encuesta recogió las percepciones de los estudiantes en relación a la metodología aplicada en las clases (tradicional o TBL). Este apartado incluyó nueve afirmaciones evaluadas según escala con cinco alternativas que van desde "Siempre" hasta "Nunca".

La Tabla 2 presenta estadísticas descriptivas de la información recopilada anónimamente para las variables utilizadas. En cada caso, se muestran los resultados obtenidos según la metodología tradicional y con TBL. El coeficiente Alpha de Cronbach estimado para la encuesta fue de 0.83 , de modo que se considera que ésta fue fiable o consistente (Cronbach, 1951; Tavakol y Dennick, 2014).

La variable Nota Final representa el promedio de la calificación final obtenida por cada uno de los grupos, y será la variable a utilizar para probar la Hipótesis 1.

La Hipótesis 2 se probará utilizando las nueve variables indicadas en la tabla, desde la A hasta la I. La escala de evaluación utilizada para estas variables va desde $5=$ siempre; 
4=casi siempre; $3=$ a veces; $2=$ casi nunca; hasta $1=$ nunca. En la tabla se puede apreciar que el promedio de las nueve variables observadas bajo la metodología TBL es mayor en todos los casos, con valores por sobre 4,0, mientras que en los estudiantes que trabajaron bajo la metodología tradicional, los valores promedio están en el rango de 3,3 y 3,9. De hecho, el promedio de las percepciones de las nueve variables bajo TBL arroja un promedio de 4,3, mientras que bajo la metodología tradicional únicamente de 3,5. Además, la variable con mayor dispersión, bajo ambas metodologías, se da en la percepción del aporte de los ayudantes en el proceso de aprendizaje.

La variable NEM_cualitativo muestra el promedio de la Nota de Enseñanza Media (NEM) obtenida por los estudiantes, definida en base a los siguientes rangos: 1 representa NEM de 4,0 a 5,0; 2 es un NEM de 5.1 a 6,0 y 3 es un NEM es de 6.1 a 7,0. Esta variable es la que se usará para la prueba de la Hipótesis 3. Se puede apreciar que el valor medio, tanto para los estudiantes que trabajaron con la metodología tradicional como con TBL, es prácticamente igual: 1,9, y que la dispersión es bastante similar: 0,72 para el primer grupo y 0,68 para el segundo grupo.

Tabla 2. Estadísticas Descriptivas de las variables consideradas en el estudio

\begin{tabular}{|c|c|c|c|c|c|c|c|c|c|}
\hline \multirow[t]{2}{*}{ Variable* } & \multirow[t]{2}{*}{ Significado } & \multicolumn{4}{|c|}{ 1-Metodología Tradicional } & \multicolumn{4}{|c|}{ 2-Metodología TBL } \\
\hline & & Mínimo & Máximo & Media & $\begin{array}{c}\text { Desv. } \\
\text { Estánd. }\end{array}$ & Mínimo & Máximo & Media & $\begin{array}{l}\text { Desv. } \\
\text { Estánd. }\end{array}$ \\
\hline NF & $\begin{array}{l}\text { Calificación obtenida } \\
\text { (Nota Final) }\end{array}$ & 4,00 & 5,80 & 4,79 & 0,62 & 4,70 & 6,20 & 5,35 & 0,45 \\
\hline NEM & $\begin{array}{l}\text { Promedio de Notas de } \\
\text { Enseñanza Media (NEM) }\end{array}$ & 1,00 & 3,00 & 1,87 & 0,72 & 1,00 & 3,00 & 1,89 & 0,68 \\
\hline Metodo & $\begin{array}{l}\text { Variable dummy: curso } \\
\text { con TBL o no. }\end{array}$ & 1,00 & 2,00 & 1,37 & 0,49 & & & & \\
\hline A & $\begin{array}{l}\text { Facilitamiento del } \\
\text { aprendizaje. }\end{array}$ & 1,00 & 5,00 & 3,65 & 0,98 & 3,00 & 5,00 & 0,44 & 0,62 \\
\hline $\mathrm{B}$ & $\begin{array}{l}\text { Estimulación de la } \\
\text { participación activa. }\end{array}$ & 1,00 & 5,00 & 3,32 & 1,22 & 2,00 & 5,00 & 4,39 & 0,85 \\
\hline $\mathrm{C}$ & $\begin{array}{l}\text { Interés en facilitar la } \\
\text { enseñanza aprendizaje. }\end{array}$ & 1,00 & 5,00 & 3,32 & 1,08 & 3,00 & 5,00 & 4,50 & 0,62 \\
\hline $\mathrm{D}$ & $\begin{array}{l}\text { Grado de utilidad del } \\
\text { material de apoyo. }\end{array}$ & 2,00 & 5,00 & 3,84 & 0,86 & 3,00 & 5,00 & 4,78 & 0,55 \\
\hline $\mathrm{E}$ & $\begin{array}{l}\text { Actividades en el tiempo } \\
\text { apropiado. }\end{array}$ & 2,00 & 5,00 & 3,94 & 0,85 & 3,00 & 5,00 & 4,56 & 0,62 \\
\hline $\mathrm{F}$ & $\begin{array}{l}\text { Se reflejan los objetivos de } \\
\text { aprendizaje. }\end{array}$ & 3,00 & 5,00 & 3,81 & 0,75 & 4,00 & 5,00 & 4,72 & 0,46 \\
\hline G & Retroalimentación fue útil. & 1,00 & 5,00 & 3,61 & 1,09 & 2,00 & 5,00 & 4,17 & 0,99 \\
\hline $\mathrm{H}$ & $\begin{array}{l}\text { Los ayudantes facilitaron } \\
\text { el aprendizaje. }\end{array}$ & 1,00 & 5,00 & 3,29 & 1,44 & 1,00 & 5,00 & 4,00 & 1,08 \\
\hline I & $\begin{array}{l}\text { El nivel de exigencia ha } \\
\text { sido apropiado. }\end{array}$ & 1,00 & 5,00 & 3,55 & 1,18 & 4,00 & 5,00 & 4,67 & 0,49 \\
\hline
\end{tabular}

*El número de observaciones es 31 en el grupo con método tradicional, y 18 en el grupo TBL. Para la variable Método son 49 observaciones.

Fuente: Elaboración propia. 


\section{RESULTADOS}

\subsection{DIFERENCIAS EN LA NOTA FINAL CON AMBAS METODOLOGÍAS}

La Hipótesis 1 plantea que la metodología TBL logra mejores resultados, es decir una nota final de aprobación de la asignatura mayor que con la metodología de cátedra tradicional. Para medir las diferencias en la nota final, se utilizó la prueba U de Mann-Whitney, una versión no paramétrica de la habitual prueba t de Student de diferencia de medias para dos muestras independientes. Para todos los casos se usó un nivel de significancia de 5\%, una estimación asintótica para el p-value, y una corrección de continuidad.

La Tabla 2 muestra que la nota final promedio de ambos grupos fue 5,35 versus 4,79, a favor del grupo con TBL. Los resultados de la prueba de Mann-Whitney a dos colas (Tabla 3) permiten rechazar la hipótesis nula, de modo que hay diferencias estadísticamente significativas en el promedio de notas finales obtenidas por ambos grupos, y se concluye que los estudiantes que trabajaron con la metodología con TBL obtienen mejores calificaciones en promedio que los estudiantes que desarrollan la asignatura a través del método tradicional.

Tabla 3. Resultados de la Hipótesis 1: Test de Mann-Whitney para la diferencia de medias de la variable "Nota Final".

\begin{tabular}{|l|c|}
\hline Item & Valor \\
\hline U de Mann-Whitney & 414,00 \\
\hline Valor Esperado & 279,00 \\
\hline Varianza (U) & $2.309,10$ \\
\hline p-value(Two-tailed) & $0,0051^{*}$ \\
\hline Alpha & 0,0500 \\
\hline
\end{tabular}

(*) Indica que el estadístico es significativo al $5 \%$.

Fuente: Elaboración propia.

\subsection{DIFERENCIAS DE PERCEPCIÓN CON AMBAS METODOLOGÍAS}

La Hipótesis 2 indica que los estudiantes tienen una mejor percepción de la metodología TBL, como herramienta de aprendizaje, sobre la metodología tradicional. Para comprobar dicha hipótesis, al igual que el caso anterior, se utilizó la prueba U de Mann-Whitney de diferencia de medias a dos colas y los resultados se muestran en la Tabla 4. 
Tabla 4. Resultados Hipótesis 2: Test de Mann-Whitney para la diferencia de medias en las variables que miden la percepción de los estudiantes

\begin{tabular}{|l|c|c|c|}
\hline & $\mathrm{U}$ & $\begin{array}{c}\text { Varianza } \\
\text { (U) }\end{array}$ & $\begin{array}{c}\text { p-value } \\
\text { (dos colas) }\end{array}$ \\
\hline A. Facilitamiento del aprendizaje. & 412,0 & 2093,6 & $0,004^{*}$ \\
\hline B. Estimulación de la participación activa. & 419,0 & 2161,4 & $0,003^{*}$ \\
\hline C. Interés en facilitar la enseñanza aprendizaje. & 451,5 & 2154,1 & $0,000^{*}$ \\
\hline D. Grado de utilidad del material de apoyo. & 446,5 & 2007,8 & $0,000^{*}$ \\
\hline E. Actividades en el tiempo apropiado. & 395,0 & 2000,3 & $0,010^{*}$ \\
\hline F. Se reflejan los objetivos de aprendizaje. & 456,5 & 2040,9 & $<0,0001^{*}$ \\
\hline G. Retroalimentación fue útil. & 364,5 & 2125,2 & 0,065 \\
\hline H. Los ayudantes facilitaron el aprendizaje. & 355,5 & 2183,8 & 0,104 \\
\hline I. El nivel de exigencia ha sido apropiado. & 441,0 & 2085,0 & $0,000^{*}$ \\
\hline
\end{tabular}

Nota: El valor esperado en todas las series fue de 279,0.

(*) Indica que el estadístico es significativo al $5 \%$.

Fuente: Elaboración propia.

En primer lugar, respecto a la metodología aplicada en clase, los estudiantes en promedio tienden a coincidir en que la nueva metodología facilitó el aprendizaje. Puesto que los estudiantes dedican más tiempo a la asignatura bajo el nuevo método, este mayor esfuerzo se vio reflejado en un mejor aprendizaje global con TBL que con la metodología tradicional, considerando el promedio de nota final de la asignatura.

También se aprecia una diferencia significativa en la opinión de que la clase con TBL aumentó la participación de los estudiantes. En efecto, en la nueva metodología los estudiantes discuten activamente los temas que se trabajan, ya sea en cada clase junto con sus compañeros de grupo o en la discusión global final de todos los estudiantes con el profesor. Este cambio es percibido como significativo y favorable por parte de los estudiantes.

En tercer lugar, los estudiantes consideran que el nuevo método implica un aprendizaje más continuo en el tiempo y más ordenado, ya que el estudio se realiza prácticamente clase a clase, de modo que consideraron que la metodología facilitó el aprendizaje. En lugar de preparar grandes cantidades de materia en poco tiempo, como lo es en el caso de la metodología tradicional, ahora deben preparar módulos más breves y precisos y esto, naturalmente, es visualizado por los estudiantes como útil para su proceso de aprendizaje.

Por otra parte, los estudiantes también consideraron que el material de apoyo del curso fue significativamente mejor con la metodología TBL. En efecto, existe ahora una mayor relación entre el material entregado cada semana y el test que se realiza sobre ese mismo material. Ahora los estudiantes aprecian una correlación más clara entre el material estudiado y la evaluación. En contraste con el método tradicional, el profesor entrega gran cantidad de material, el cual es evaluado muy parcialmente en una prueba global, y puede esperarse que los estudiantes intenten extraer en breve tiempo cuales son los aspectos más 
importantes de dicho material. En TBL la mayor parte del material entregado es relevante, pues este se remite como máximo a un capítulo o una parte de capítulo, y un pequeño número de problemas resueltos.

Respecto del uso del tiempo, los estudiantes también consideran que las actividades se desarrollaron y se ajustaron a un cronograma de tiempo más apropiado. Esto se explica por el hecho de que con TBL se espera que administren mejor sus tiempos de estudio, sin sentirse agobiados por la necesidad de preparar grandes cantidades de materia en breves lapsos de tiempo, como lo exige la metodología tradicional.

Otra observación fue que los estudiantes consideraron que la calificación conseguida con el nuevo método se ajusta mejor al aprendizaje alcanzado. Por una parte, esto se explica por el hecho de que se realiza un número mucho mayor de evaluaciones, y con mucha mayor frecuencia que bajo el modelo tradicional. Se espera que ahora los estudiantes aprendan de sus errores y logren optimizar sus esfuerzos con mucha mayor rapidez, esto por medio de la retroalimentación continua. En el caso tradicional, el tiempo destinado a evaluar las asignaturas en conjunto puede aproximarse a unas 5 o 6 horas (dos pruebas parciales y un examen) en un semestre. Sin embargo, con TBL el tiempo destinado a evaluación supera las 15 horas en total. De esta forma, se obtiene un muestreo de evaluaciones más representativo del verdadero grado de aprendizaje de los estudiantes.

Respecto a la retroalimentación, los estudiantes no consideran que hubo un cambio significativo entre ambas metodologías. Esto es razonable, por cuanto en el método tradicional el profesor juega un papel activo en esta parte, pues es él quien desarrolla y explica a los estudiantes paso a paso la pauta de las pruebas. En el TBL, el profesor juega un papel secundario en esta parte, por cuanto son los propios estudiantes los que se retroalimentan. En efecto, cuando han respondido el test individual generalmente mantienen dudas respecto a las respuestas correctas, pero cuando se pasa a la fase grupal, la discusión al interior de cada grupo les permite generalmente aclarar autónomamente la casi totalidad de las dudas, sin que el profesor juegue un papel principal aquí. La experiencia mostró que menos de un $20 \%$ del test requería algún grado de revisión con el profesor en la discusión final y, generalmente, esto ocurrió con las preguntas de tipo cualitativas, debido a que la forma en que las preguntas estaban redactadas fue interpretada de diferente modo por parte de los estudiantes. Fue muy raro que problemas cuantitativos requirieran una discusión grupal posterior con el profesor.

Ante la pregunta de si los ayudantes facilitaron el aprendizaje, nuevamente los estudiantes consideraron que no hubo un cambio significativo entre ambas metodologías (la hipótesis nula no pudo ser rechazada ( $\mathrm{p}=0,104)$. Esto parece explicarse por el hecho de que la nueva metodología se basa en un autoaprendizaje, en que es el propio estudiante y sus compañeros de grupo quienes descubren el conocimiento y se retroalimentan entre sí, quedando en el TBL el ayudante relegado a un papel menos activo. El papel tradicional del ayudante era preparar ayudantías y material de apoyo, resolviéndolo en un horario de clases de ayudantía. Esto ahora ya no ocurre, y el ayudante dedica la mayor parte del tiempo a colaborar con el profesor en preparar los test semanales, y test de apoyo. Puesto que todo se hace con alternativas de selección múltiple, esto requiere bastante trabajo en plantear y resolver un gran número de problemas para finalmente seleccionar los que se consideren apropiados para el test. Luego, dado el nuevo papel que juegan los ayudantes con la nueva metodología, es comprensible que los estudiantes no perciban que en la nueva metodología los ayudantes faciliten el aprendizaje. 
Respecto al nivel de exigencia de las evaluaciones del curso, los estudiantes consideran que la nueva metodología logra ajustar mejor la exigencia, debido justamente al hecho de que ahora la carga de trabajo de la asignatura se distribuye de un modo más homogéneo durante el curso, lo que es más cómodo para los estudiantes. Esto lo diferencia del método tradicional en que existen períodos de muy baja y muy alta actividad. Otro aspecto que conviene destacar, es el hecho de que la asistencia a clases en el nuevo método resultó extraordinariamente alta, debido a la necesidad de los estudiantes de rendir los test periódicos. Esta mayor asistencia a clases se espera que se traduzca en menores vacíos de conocimiento a ser resueltos para un examen final de la asignatura.

Como se puede notar, las apreciaciones anteriores de los estudiantes permiten concluir que, en general, los estudiantes valoran positivamente la implementación de la nueva técnica, tanto por una mejoría en la metodología aplicada, como por un mayor grado de aprendizaje, mayor participación, mejor material, una mejor metodología de evaluación y exigencias más apropiadas en las evaluaciones.

\subsection{APORTE COMPARATIVO DE TBL Y NEM SOBRE LA NOTA FINAL CON AMBAS METODOLOGÍAS}

Generalmente se señala que el promedio de las Notas de Enseñanza Media (NEM) de los estudiantes son un buen predictor del desempeño de los universitarios y, efectivamente, esto ha sido apoyado por otros estudios en Chile (véase Soria-Barreto y Zúñiga-Jara, 2014). Para completar esta afirmación, la Hipótesis 3 se plantea un paso más en la aportación de las notas NEM y del método empleado, incorporando la relación conjunta de ambas variables sobre la nota final. En principio, se consideraba que mayores NEM, combinadas con el empleo de TBL, iban a favorecer el aprendizaje de los estudiantes.

Para la verificación de esta hipótesis se formula un ANOVA de dos factores, con varias muestras por grupo, en el que se postula que la nota de aprobación de la asignatura es explicado por dos variables: (1) la implementación de la técnica de TBL y, simultáneamente, por las NEM; (2) categorizadas en tres grupos (alto, medio, y bajo NEM).

La Tabla 5 permite apreciar que se refuerza la existencia de un efecto significativo de la técnica TBL en la nota final $(\mathrm{p}=0.004)$. Sin embargo, esta relación positiva no se va a repetir en el caso de las NEM, ya que el p-value muestra que éstas no tienen un efecto distinguible en la calificación final. Este resultado contrasta con el obtenido en otros estudios recientes, como el de Soria-Barreto y Zúñiga-Jara (2014), quienes encontraron que la principal variable explicativa del éxito en el egreso de la carrera universitaria eran las notas de enseñanza media. Sin embargo, debido a que el éxito en el egreso ha sido generalmente medido a través de la metodología tradicional, es posible que justamente la metodología tradicional se ajuste mejor para estudiantes disciplinados, es decir los que tienen NEM más altos. Puesto que los resultados de la Tabla 5 indican que no existió diferencia en los resultados en notas finales del curso para ambos grupos, los resultados sugieren entonces que el TBL logra nivelar positivamente a los estudiantes de menores NEM, haciendo indistinguibles las diferencias en sus NEM respecto al resultado final. Estudios posteriores sobre este punto permitirán a futuro clarificar mejor esta importante interrogante.

Adicionalmente, la tabla muestra que tampoco existe un efecto de interacción entre las técnicas de enseñanza y el hecho de obtener altas o bajas NEM relativas (el p-value de Método*NEM no es representativo), de modo que aplicando las dos variables 
simultáneamente en el modelo, conjuntamente no resultan explicativas de la nota final. Luego se confirma aquí el principal resultado del estudio, que las notas de aprobación al curso sí se ven afectadas por el método de enseñanza (con o sin TBL), pero sin ser afectadas por las NEM.

Tabla 5. Resultados Hipótesis 3: ANOVA para el efecto en la calificación final (NF) del Método docente utilizado (Método) y de las notas de enseñanza media (NEM)

\begin{tabular}{|l|c|c|c|c|c|}
\hline \multicolumn{1}{|c|}{ Fuente } & DF & Suma de Cuadrados & Cuadrado de Medias & F & Pr $>$ F \\
\hline Metodo & 1 & 2,760 & 2,760 & 9,084 & $0,004^{*}$ \\
\hline NEM & 2 & 1,090 & 0,545 & 1,795 & 0,178 \\
\hline Metodo*NEM & 2 & 0,079 & 0,040 & 0,130 & 0,878 \\
\hline Error & 43 & 13,063 & 0,304 & & \\
\hline
\end{tabular}

Método=Variable dicotómica que indica pertenencia al grupo que usó el método tradicional (Método=1), o al grupo que usó TBL (Método=2)

Método*NEM=Variable que mide el efecto de interacción conjunta entre el Método y las notas de enseñanza media.

* Indica que el estadístico es significativo al 5\%.

Fuente: Elaboración propia.

\section{CONCLUSIONES}

En este estudio se analizó el efecto de la implementación de nuevas metodologías de aprendizaje en una muestra de estudiantes universitarios, comparando dos grupos que recibieron la misma materia, pero a través de dos metodologías diferentes (TBL y Tradicional).

Como resultado del estudio se puede concluir que la aplicación de esta nueva metodología no sólo generó un incremento significativo en la nota final de la asignatura, sino que también formó una opinión más favorable del estudiante hacia la asignatura. El cambio supuso una mejora en la percepción en la mayor parte del alumnado acerca de la facilidad del aprendizaje, indicando que, en comparación al método clásico de la clase magistral, con el TBL se estimuló la participación activa en las clases, el material de apoyo fue más útil para el estudio, los objetivos de aprendizaje se lograron mejor, y el nivel de exigencia fue más adecuado. Por otro lado, el mejor resultado del curso generado por el método TBL no se explica por las variables Notas de Enseñanza Media (NEM) de los estudiantes (una proxy de buenos métodos de estudio en los estudiantes de enseñanza media), sino que se explica casi completamente por el hecho de aplicar la nueva metodología de enseñanza. Este resultado no coincidió con lo que inicialmente se podía esperar, y contrasta con los resultados de otros estudios recientes (Soria-Barreto y ZúñigaJara, 2014), que identificaron a la NEM como principal variable explicativa de la nota final en cursos basados en las metodologías tradicionales.

Esta experiencia de empleo de una metodología en la que la clase deja de ser magistral y pasa a tener una estructura diferente (test individual - test grupal con empleo IF-AT 
- retroalimentación - clase expositiva - preparación individual de la siguiente sesión), puede ser considerada como positiva por parte de los estudiantes, quienes parecen haber podido desarrollar también capacidades como la autorresponsabilidad, trabajo en equipo, la exposición de ideas ante sus pares, y la organización autónoma del trabajo. Por todo esto, parece interesante el plantearse la extensión de esta técnica de enseñanza-aprendizaje a otros ramos de las carreras universitarias.

Finalmente, los resultados de este estudio proporcionan interesante evidencia de que la metodología del TBL puede ser implementada en cursos numerosos, con trabajo en subgrupos reducidos, y logrando una docencia centrada más en el estudiante que en el profesor, sin precisar grandes recursos de infraestructura. En efecto, las necesidades a este respecto se limitan a salas con distribución flexible, donde las mesas se puedan mover para facilitar la relación de los estudiantes que forman cada grupo, y algunos requerimientos técnicos básicos adicionales. El profesor pudo procesar la información de forma parecida a la de un curso tradicional, pero encargando a los estudiantes la resolución de problemas o casos determinados, formando para ello equipos. Globalmente, los resultados de este estudio suponen un incentivo adicional para incorporar el empleo de nuevas metodologías cada vez más activas en la enseñanza universitaria.

\section{BIBLIOGRAFÍA}

Abbott, L. (2012). Tired of Teaching to the Test? Alternative Approaches to Assessing Student Learning. Rangelands, vol. 34, 34-38.

Angelo, T. y Cross, K. P. (1993). Classroom assessment techniques. A handbook for college teachers. San Francisco: Jossey-Bass Publishers

Asún,I.; R., Zúñiga, C. y Ayala, M. C. (2012). La formación por competencias y los estudiantes: confluencias y divergencias en la construcción del docente ideal. Calidad en la educación, vol. $38,277-304$.

Brodbeck, F., Kerschreiter, R., Mojzisc, A., Frey, D. y Schulz-Hardt, S. (2002). The dissemination of critical, unshared information in decision-making groups: the effects of pre-discussion dissent. European Journal of Social Psychology, vol. 32, 35-56.

Celina, H. y Campo A. (2005). Aproximación al uso del coeficiente Alfa de Cronbach. Revista Colombiana de Psiquiatría, vol. 34, n. 4, 572-580.

Chain, R., N. Cruz, N., Martínez, M. y Jácome, N. (2003). Examen de Selección de Probabilidad de Éxito Escolar en Estudios Superiores. Estudio en una universidad pública estatal mexicana: Revista Electrónica de Investigación Educativa, vol. 5(1), n. 17.

Clark, M. C.; Nguyen, H. T., Bray, C. y Levine, R. E. (2008). Team Based Learning in an Undergraduate Nursing Course. The Journal of Nursing Education, vol. 47, 111-117.

Cronbach, L. J. (1951). Coefficient Alpha and the Internal Structure of Tests. Psychometrika, vol. $16,1-16$.

Duarte, M. y J. Galaz, J. (2006). Predicciones del desempeño académico en el $1^{\circ}$ año de universidad en una institución pública estatal (Universidad Autónoma de Baja California). Trabajo preparado para el " 6 Congreso Internacional Retos y Expectativas de la Universidad: El Papel de la Universidad en la Transformación de la Sociedad, Puebla, México

Epstein, J. (1989). Family structures and student motivation: A developmental perspective. In C. Ames \& R. Ames (Eds). Research on motivation in education, vol. 3. Nueva York: Academic Press

Lazerson, M., Wagener, U. y Shumanis, N. (1999). What Makes a Revolution: Teaching and Learning 
in Higher Education, 1980-2000. National Center for Postsecondary Improvement. Consultado en junio de 2014, www.stanford.edu/group/ncpi/documents/pdfs/5-11_revolution.pdf

Lee, M.; Mella, J.; Ocaranza, D. y Lagos, K. (2014). Team based learning and immediate feedback assessment technique. Jornadas de Educación XIV en Ciencias de la Salud. Universidad de Chile

Lerner, J. S. y Tetlock, P. E. (1999). Accounting for the effects of Accountability. Psychological Bulletin, vol. 125, 255-275.

Michaelsen, L. y Sweet, M. (2008). The Essential Elements of Team-Based Learning, in New Directions for Teaching and Learning. Special issue: Team-Based Learning: Small Group Learning's Next Big Step, vol. 116, 7-27.

Michaelsen, L., Bauman, A. y Fink, L. (2002). Team-Based Learning: A Transformative Use of Small Groups in New Directions for Teaching and Learning. Connecticut: Praeger Publishers

Moreno, T. (2010). El currículo por competencias en la universidad: más ruido que nueces. Revista de la educación superior, vol.39, 77-90.

Nieder, G., Dean, X. P., Stolfi, A. y Hudes, P. (2005).Team-Based Learning in a Medical Gross Anatomy and Embryology Course. Clinical Anatomy, vol. 18 (1), 56-63.

Polimeno, N. C., Duarte, M., Dotta, M., Scorsoline, A., Jarochinski Silva, J. C., Carvalho, D. C. y Medeiros, J. (2012). A Estratégia da Aprendizagem Baseada em Equipes em um Curso de Direito. Revista de Educación y Derecho (Education And Law Review), vol. 7.

Salgado, F.; Corrales, J.; Muñoz, L. y Delgado, J. (2012). Diseño de programas de asignaturas basados en competencias y su aplicación en la Universidad del Bío-Bío, Chile. Ingeniare. Revista chilena de ingeniería, vol. 20, n. 2, 267-278.

Soria-Barreto, K. y Zúñiga-Jara, S. (2014). Determinantes de Éxito Académico en Estudiantes Universitarios. Por aparecer en Revista Formación Universitaria.

Tavakol, M. y Dennick, R. (2011). Making sense of Cronbach's Alpha. International Journal of Medical Education, vol. 2, 53-55. 
